\section{SAFETY PERFORMANCE EVALUATION AND ACCIDENT PREVENTION USING PROACTIVE INDICATORS: A CASE STUDY OF AN INDUSTRY}

${ }^{1}$ Fatemeh aminifard*, ${ }^{2}$ Shahram mahmoudi, ${ }^{1}$ Ali Kianfar. ${ }^{1}$ Health, safety and environment specialist in MAPNA Group, Tehran, Iran; ${ }^{2}$ Health, safety and environment manager in MAPNA Group, Tehran, Iran

\subsection{6/oemed-2018-ICOHabstracts.80}

Introduction Quality and effectiveness of safety systems are critical factors in achieving their goals. This study was aimed to represent a method to performance evaluation of Safety systems by proactive indicators using different updated models in the field of safety which will be tested in a selected industry in order to prevent accidents and injuries from occurring in the future.

Methods This study is a cross-sectional study. Proactive indicators used in this study were: Unsafe acts rate, Safety Climate, Accident Proneness and Near-miss incidents rate.

Results The number of in 1473 safety climate questionnaires and 543 Accident Proneness questionnaires were completed. The minimum and maximum safety climate score were 56.88 and 58.2, respectively and the minimum and maximum scores of Accident Proneness were 98.2 and 140.7 respectively. The maximum number of Near-miss incidents rate were 408 and the minimum of that was 196. The maximum number of unsafe acts rate was 43.8 percent and the minimum of that was 27.2 percent. In nine dimensions of Safety climate the eighth (personal perception of risk) with 4.07 has the lowest score and the fourth (laws and safety regulations) with 8.05 has the highest score. According to experts opinions the most important indicator in the assessment of safety performance was 'unsafe acts rate' and 'Near-miss incidents rate' was the less importance. The study finds significant correlation between unsafe acts rate and number of lost work day's accidents.

Discussion The results of this survey reveal that using proactive (Prospective) indictors could be an appropriate method in organisations safety performance evaluation and sequentially preventing accidents from occurring.

\section{EPIDEMIOLOGICAL SURVEILLANCE OF WORK-RELATED INJURIES IN NORWAY: PERVASIVE CHALLENGES AND MISSED OPPORTUNITIES?}

${ }^{1}$ YS Samant ${ }^{*},{ }^{2} \mathrm{HM}$ Gravseth, ${ }^{1} \mathrm{TE}$ Danielsen. 'Norwegian Labour Inspection Authority, Norway; ${ }^{2}$ National Institute of Occupational Health, Norway

\subsection{6/oemed-2018-ICOHabstracts.81}

Objectives Data on work-related injury is critical in devising preventive strategies. In Norway, there are different systems that yield epidemiological data on work-related injuries, both fatal and non-fatal. This is a comprehensive profile of surveillance of work-related injuries in Norway. Moreover, we attempt to highlight the challenges and missed opportunities with regards to improving surveillance, and thereby prevention of work-related injuries.

Methods We collated information from several Norwegian studies that evaluated the different systems that yield epidemiological data on work-related injuries. These studies identified several challenges with regards to injury data collected by different institutions like the Labour Inspection, Public Health Institute, Registry of Private Insurance Companies and Hospital Based Registry. Several public documents that concern national strategies for improving work-related injury surveillance in Norway were also examined.

Results None of the injury surveillance systems provided an accurate representation of work-related injuries. However, it is fair to submit that surveillance of work-related fatal injuries has improved in the last few years. Unfortunately, the same cannot be said about non-fatal traumatic injuries, and unintentional injuries attributed to work. Our findings indicate several challenges with regards to the surveillance infrastructure. These challenges could be attributed to among others, underreporting, unclear reporting criteria, lack of coordination between the national agencies, tenuous quality assurance and inapt use of available technologies.

Conclusions It must be said that significant gains have been made in the past few years with regards to fatal injury surveillance, although improvement on evaluation of completeness of captured data and quality assurance is much desired. However, the surveillance of non-fatal injuries remains an enduring challenge.

There are both sound policy-level, and novel technological opportunities available for improving the current situation. But, these opportunities have been missed thus far. Consequently, challenges to surveillance of work-related injuries remain pervasive limiting our preventive efforts.

\section{UTILITY OF STABILOMETRIC FOOTBOARD IN PREVENTION OF WORK ACCIDENT FROM FALL. AN ITALIAN STUDY IN A POPULATION OF AT-HEIGHT WORKERS}

${ }^{1}$ Marco Italo D'Orso, ${ }^{1}$ Michele Augusto Riva, ${ }^{2}$ llaria Invernizzi, ${ }^{3}$ Daniele Grosso, ${ }^{1}$ Giancarlo Cesana. 'University Of Milan Bicocca, Monza, Italy; ${ }^{2}$ Consortium for the development of Occupational and Environmental Medicine, Monza, Italy; ${ }^{3}$ Synlab Italia S.r.l., Monza, Italy

\subsection{6/oemed-2018-ICOHabstracts.82}

Introduction Precipitations at workplaces still are a frequent cause of severe or mortal work accidents in Italy. Evaluation of at-height work suitability is by now carried out using many different medical clinical protocols usually based on clinical otorhinolaryngoiatric visit. This visit is frequently carried out with clinical tests the evaluation of which is merely subjective and therefore not always comparable.

Methods To verify the possibility of increasing accuracy of evaluation of at-height workers and to better point out workers hyper susceptible to fall, we experimentally insert in our usual clinical otorhinolaryngoiatric protocol an instrumental balance evaluation carried out using a stabylometric computerised footboard (ARGO Balance Static Force Platform RGMB) collecting contemporaneously 27 items.

The clinical specialised otovestibular examination was composed by a specialised anamnesis, an otorhinolaryngoiatric medical examination, evaluation of tonic segmentary deviations, Romberg Test, research of spontaneous nystagmus, Barany test, Weits test.

We compared the results of clinical and instrumental examination in 2.082 male workers aged from 18 to 65 years engaged in at-height work.

Results 1.775 of patients included in the research have been defined as suitable for at-height work after the otorhinolaryngoiatric clinical evaluation carried out in an Occupational Health Medical Surveillance. Among them 93 had pathological results at footboard. This fact originates after six months in these workers a second clinical and instrumental examination which in 12 cases has motivated a change in workers' specific 